\title{
A Unicast Retransmission Scheme based on Network Coding
}

\author{
Jawad Manssour, Afif Osseiran, and Slimane Ben Slimane, Member, IEEE
}

\begin{abstract}
A novel scheme for data retransmission for wireless unicast communication is presented. The scheme is based on a transmitter and receiver structure and bit-level data processing using a combination of channel coding and network coding that allows retransmissions to contain the previously incorrectly received information and new information, both destined to the same receiver. Results show that, for the chosen forward error codes, up to $68.75 \%$ retransmission throughput gains are achieved compared to HARQ with Chase combining.
\end{abstract}

\section{Key Words: Forward Error Correction, HARQ, Network Coding.}

\section{INTRODUCTION}

Transmissions over wireless channels are subject to errors mainly due to the unreliable nature of the wireless medium. One way to overcome this unreliability is through the usage of link adaptation that provides a way of overcoming the fluctuations in the received signal. However, receiver noise and unpredictable variations in interference make it all the more tougher that link adaptation will suffice to have a robust transmission. One additional way to increase the robustness is through the usage of retransmissions based on error detection as done in Automatic Repeat reQuest (ARQ) schemes, or further utilizing forward error correction (FEC) resulting in Hybrid ARQ (HARQ) schemes. In order to exploit the information from the different (re)transmissions, HARQ is typically used in conjunction with soft combining and works as follows.

Jawad Manssour is with the LTE System Management group, Ericsson AB, Stockholm, Sweden (email: Jawad.Manssour@ericsson.com).

Afif Osseiran is with Ericsson Research, Stockholm, Sweden (email: Afif.Osseiran@ericsson.com.

Slimane Ben Slimane is with the Radio Communication Systems group, department of Communication Systems, the Royal Institute of Technology (KTH), Stockholm, Sweden (email: slimane@ radio.kth.se).

Copyright (c) 2011 IEEE. Personal use of this material is permitted. However, permission to use this material for any other purposes must be obtained from the IEEE by sending a request to pubspermissions@ieee.org.
When the receiver fails to decode a certain packet, it sends a negative acknowledgment (NACK) to the transmitter who in turn re-sends that packet. The receiver then combines the packet that was not correctly decoded with subsequent retransmission(s) of that same packet, resulting in a combined packet that is more reliable to decode than any of its constituents. Two variants of HARQ with soft combining exist: Chase combining (CC) [1] and incremental redundancy (IR) [2]. In the case of $\mathrm{CC}$, the retransmitted packet is identical to the original transmission. The receiver uses maximum ratio combining (MRC) to combine the received bits with the same bits from previous transmissions. With IR, each retransmitted packet includes new redundancy bits produced by the channel encoder. The decoder then combines all the transmissions and decodes the packet at a lower equivalent code rate. It shall be noted that IR provides very little gains compared to $\mathrm{CC}$ when a robust link adaptation is used [3].

\section{A. Existing Solutions}

To improve the system efficiency, the use of network coding (NC) with retransmissions has been proposed for multi-unicast flows [4] and wireless broadcasting [5] (i.e. point-to-multipoint communication). In these schemes, packets lost by different nodes (i.e. users) are combined e.g. through bitwise XOR, into one network coded packet, leading to a reduction in the total number of required retransmissions from a system perspective. This decrease in the number of retransmissions would then lead to a throughput gain compared to retransmitting each packet separately. However, from an end-user perspective, the fundamental principle of operation is still similar to conventional retransmissions: a retransmission will consist of bits that represent the information that the receiver failed to correctly decode after the initial transmission, i.e. no new 
information is transmitted during a retransmission phase.

On the other hand, the application of NC to single unicast retransmissions where the communication takes place between two nodes (i.e. point-to-point communication where the retransmission contains information destined to only one node) has been much less investigated. For instance, only recently have Li et al. [6] and Yun et al. [7] suggested to exploit NC for unicast transmissions. [6] proposed to combine multiple packets that were erroneously received into a single transmission using linear network coding [8] in order to improve the throughput. In [7] it is suggested to combine, at a symbol level, a new packet with each retransmitted packet.

\section{B. Contribution and Goal of this Work}

In this work, we present a novel scheme for retransmitting data for wireless unicast communication where a retransmission would not only contain the retransmitted data, but also new data. We propose a transmitter/receiver structure and data processing that enable such an approach based on a combination of channel coding and network coding. The retransmitted bits are conveniently combined with new bits at the transmitter, whereas the receiver exploits the incorrectly decoded data after the first transmission to separate the old and new bits received during the retransmission phase. In contrast to [7] where a similar idea was devised for the analog network coding scenario (i.e. operation on already modulated symbols), the proposed method herein doesn't alter the constellation of the used modulation hence keeping the signal space and decision boundaries for the modulation intact. Consequently the proposed method is transparent to the modulation and demodulation procedures. In addition, it is applicable to any type of modulation.

\section{System MOdEL}

In this work, we focus on a coded point-to-point communication system using linear block codes $\left(n, k, d_{\min }\right)$ where $n$ is the block (or codeword) length, $k$ is the information length and $d_{\min }$ is the corresponding minimum distance. We assume a flat fading independent and identically distributed Rayleigh channel $h_{i}$ between the transmitter and the receiver on each transmitted symbol where BPSK is utilized for simplicity. As such, the received symbol for a given symbol interval $i$ of a certain codeword is given by:

$$
r_{i}=\sqrt{P} h_{i} s_{i}+z_{i} ; \quad i=1, \ldots, n
$$

where $r_{i}$ is the received symbol sample during the transmission phase, $P$ is the transmit power per symbol, $s_{i}$ is the normalized transmitted complex symbol with $E\left\{\left|s_{i}\right|^{2}\right\}=1$ and $z_{i}$ is the complex Gaussian noise at the receiver with zero mean and double sided power spectral density $N_{0}$. In case of a retransmission, the corresponding entities are denoted with a prime $(')$ symbol, e.g. $r_{i}^{\prime}$ represents the $i$-th received retransmitted symbol. In order to distinguish symbol/bit entities from vectors, a boldface notation is used to indicate the latter. For instance, $\boldsymbol{r}$ refers to a received modulated codeword vector with length $n, \boldsymbol{h}$ and $\boldsymbol{c}$ refer to the corresponding channel vector and hard demodulated codeword, while $\boldsymbol{b}$ refers to the information vector of length $k$.

Upon reception of every codeword, the receiver makes a decoding attempt and feeds back the result to the transmitter where it is assumed that the feedback channel is error-free. In case of wrong decoding, the transmitter sends a NACK and a retransmission takes place.

\section{Proposed System Structure}

\section{A. Transmitter Structure}

The transmitter structure for the initial transmission is similar to conventional schemes: the bits are coded then modulated prior to transmission. In the remainder of this paper, the modulation (MOD) and demodulation (DEM) blocks are skipped, except when used at intermediate stages, to simplify the illustrations and focus on the main idea. On the other hand, the proposed retransmission phase will consist of combining a failed codeword (i.e. the incorrectly decoded codeword after the initial transmission) with a new codeword, both destined to the same receiver, as shown in the Retx block in Fig. 1.

The initially transmitted, but incorrectly received bits $b_{1}$ are re-encoded with the same forward error correction (FEC) code FEC 1 used in the transmission phase resulting in the same previously transmitted codeword $c_{1}$. The new information bits to be transmitted, $\boldsymbol{b}_{\mathbf{2}}$, are encoded with a code of different rate FEC 2 resulting in a codeword $\boldsymbol{c}_{2}$. 

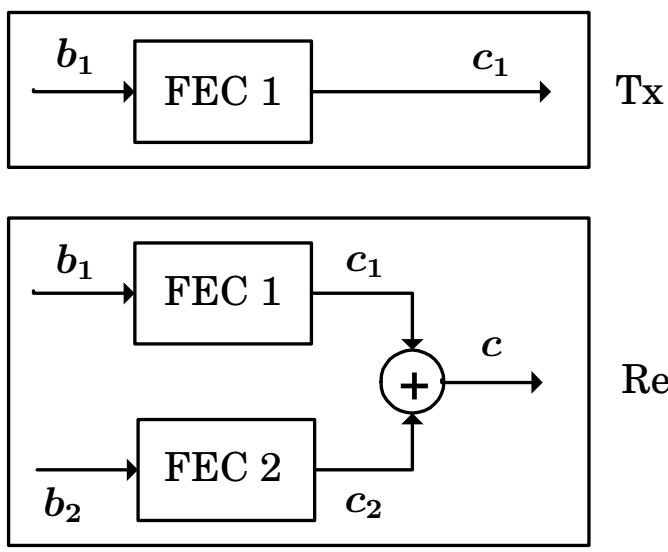

Retx

Fig. 1. The proposed transmitter structure: combining a failed and a new codeword into one equivalent codeword during the retransmission phase.

The code rate of $\boldsymbol{c}_{\mathbf{2}}$ has to be chosen lower than that of $\boldsymbol{c}_{\mathbf{1}}$, i.e. $\left(k_{2} / n_{2}\right)<\left(k_{1} / n_{1}\right)$. Having a lower rate for FEC 2 compared to FEC 1 is necessary for a proper operation of the proposed scheme and will be motivated subsequently.

Choosing FEC 2 as a subset of FEC 1 and assuming linear block codes are utilized, $\boldsymbol{c}=\boldsymbol{c}_{\boldsymbol{1}} \oplus$ $c_{2}$ (where $\oplus$ represents a bitwise XOR operation) will constitute in fact a valid codeword in FEC 1, thus possessing error correction capabilities. This has the main advantage that the receiver will not see $c_{2}$ as noise added on top of $c_{1}$, but will impose the following minor design constraint at the transmitter: $n_{2}$ has to be equal to $n_{1}$ while having $k_{2}<k_{1}$. Afterwards, $c$ is modulated and transmitted. Given an estimate of $c$ and the estimate of the failed codeword $c_{1}$ from the initial transmission phase, the receiver tries to recover $\boldsymbol{b}_{1}$ and $\boldsymbol{b}_{2}$.

\section{B. Receiver Structure}

The basic non-iterative receiver structure and data processing chain are shown in Fig. 2.

In order to recover $b_{1}$ and $b_{2}$, the receiver also makes use of the following information from the transmission phase: $\boldsymbol{r}_{\mathbf{1}}$ which contains soft information of the transmitted codeword during the transmission phase, and $\tilde{\boldsymbol{c}_{1}}$ which is the corresponding hard-demodulated codeword given by $\tilde{\boldsymbol{c}_{1}}=\boldsymbol{c}_{1} \oplus$ $e_{1}$ where $e_{1}$ is a vector representing the errors that could not be corrected by FEC 1 after the initial transmission (i.e. contains a 1 at every bit-error location).
The transmitted signal during the retransmission phase is first detected and demodulated into $\tilde{\boldsymbol{c}}$, decoded and then re-encoded resulting in the estimated $\hat{c}$ given by:

$$
\hat{\mathbf{c}}= \begin{cases}\mathbf{c} & \text { If decoding succeeded } \\ \mathbf{c} \oplus \mathbf{e}_{\mathbf{x}} & \text { If decoding failed }\end{cases}
$$

where $e_{\boldsymbol{x}}$ is a vector representing the errors in the reconstructed $c$ at the receiver. The receiver then tries to decode $c_{2}$ by first obtaining an estimate of it, $\tilde{c_{2}}$, as follows:

$$
\tilde{\mathbf{c}_{2}}=\hat{\mathbf{c}} \oplus \tilde{\mathbf{c}_{\mathbf{1}}}=\underbrace{\mathbf{c} \oplus \mathbf{c}_{1}}_{\mathbf{c}_{\mathbf{2}}} \oplus \mathbf{e}_{\mathbf{x}} \oplus \mathbf{e}_{\mathbf{1}}
$$

In order to recover $\boldsymbol{b}_{\mathbf{2}}$, FEC 2 needs to be able to correct up to $\boldsymbol{e}_{\mathbf{1}} \oplus \boldsymbol{e}_{\boldsymbol{x}}$ errors. However, if $\boldsymbol{c}$ was received correctly, FEC 2 needs to be able to correct only $e_{1}$ errors, i.e. the errors that FEC 1 could not correct after the initial transmission. This is why FEC 2 needs to always have a lower rate (i.e. be more robust) than FEC 1.

After performing DEC 2 , the estimated $\hat{b_{2}}$ are then re-encoded into $\hat{c_{2}}$ and added to $\hat{c}$ to obtain $\hat{c_{1}}$ which is an estimate of $c_{1}$ from the retransmission phase. $\hat{c_{1}}$ is then remodulated and weighted by the estimated channel $\boldsymbol{h}^{\prime}$ from the retransmission phase in order to obtain the soft information $\boldsymbol{r}_{1}^{\prime}$. The quality of $r_{1}^{\prime}$ is not only dependent on the channel quality during the retransmission phase, but also on the residual errors $\boldsymbol{e}_{2}$ resulting from the difference between $\hat{c_{2}}$ and $\boldsymbol{c}_{2} \cdot \boldsymbol{r}_{1}^{\prime}$ is then combined using MRC with $r_{1}$ and the resultant information is hard demodulated then decoded to obtain $\hat{b_{1}}$.

In addition to the basic non-iterative structure described above, the processing is complemented with one iteration where $\hat{b_{1}}$ are re-encoded using FEC 1 and then added onto $\tilde{c_{1}}$ in order to obtain an enhanced version of $\tilde{c_{2}}$. The same processing as in the basic structure is carried out in order to obtain an enhanced estimate of $r_{1}^{\prime}$, combine it with $r_{1}$ and further improve $\hat{b_{1}}$. Simulation results are subsequently provided for both non-iterative and iterative variants.

\section{Performance Measures and Simulation SETUP}

In order to evaluate the performance of the proposed scheme, its throughput is measured and compared to that of a system utilizing HARQ based 


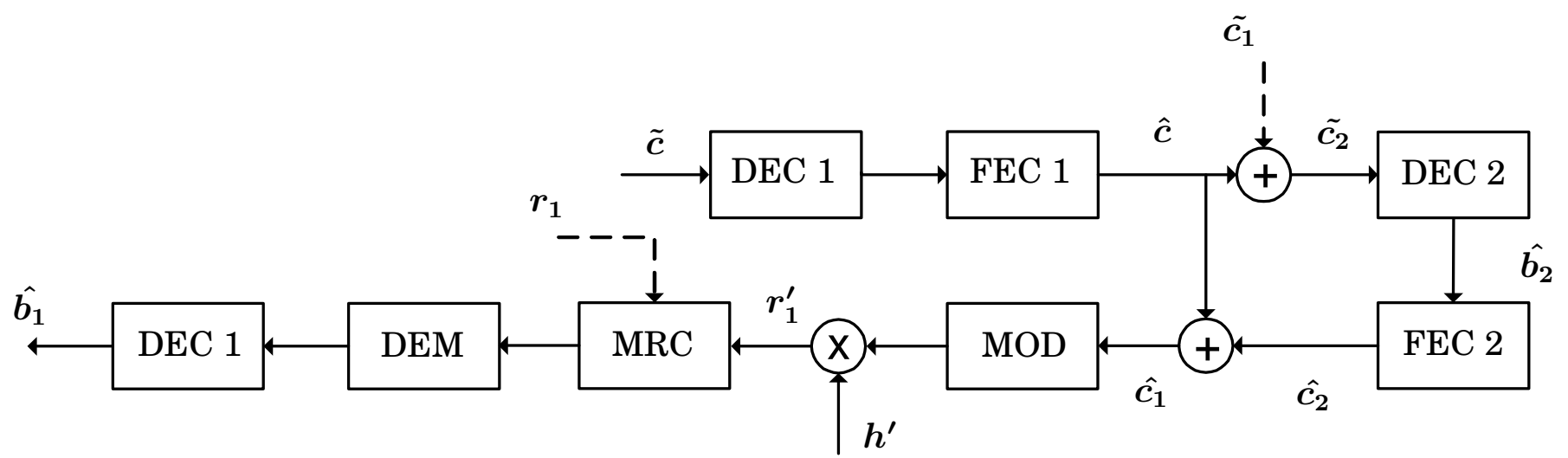

Fig. 2. The basic proposed receiver structure and data processing using one iteration. Information from the transmission phase is shown using dashed lines.

on CC. Two measures of throughput are utilized: total throughput and retransmission throughput. Total throughput is defined as the total number of bits correctly received after both the transmission and retransmission phases. Retransmission throughput is defined as the total number of bits correctly received during the retransmission phase only. As the conventional retransmission and the proposed method transmit utilizing codewords of the same size (i.e. $c$ and $c_{1}$ are of the same size), there is no need for normalization by the utilized codeword size for a fair comparison. Furthermore, as both the proposed scheme and the conventional scheme utilize the same method for the initial transmission, this would result in the same BLock Error Probability (BLEP) for $c_{1}$ after the transmission phase. The BLEP at the first transmission is typically utilized as an indication of how practical the chosen code for the initial transmission (i.e. FEC 1) is, and for what type of applications it is suitable for.

Assuming only one retransmission is allowed per failed codeword for simplicity, the total throughput when using conventional HARQ with $\mathrm{CC}$, and the proposed method, are respectively given by:

$$
\begin{gathered}
C_{\text {conv }}=E\left\{k_{1} \sum_{l=1}^{M} \pi_{l}\right\}+\underbrace{E\left\{k_{1} \sum_{j=1}^{N} \eta_{j}\right\}}_{\text {Retransmission throughput }} \\
C_{\text {prop }}=E\left\{k_{1} \sum_{l=1}^{M} \pi_{l}\right\}+\underbrace{E\left\{k_{1} \sum_{j=1}^{N} \zeta_{j}+k_{2} \sum_{j=1}^{N} \psi_{j}\right\}}_{\text {Retransmission throughput }}
\end{gathered}
$$

where $E\{$.$\} represents the expectation, M$ is the total number of codewords transmitted during the initial transmission and $N$ is the number of codewords that failed after the initial transmission and had to be retransmitted (i.e. $N \leq M$ ). $\pi_{l}$ is a variable indicating the block error event of the $l$ th $c_{1}$ where $\pi_{l}=1$ in case of correct decoding, and $\pi_{l}=0$ otherwise. Similarly, $\eta_{j}, \psi_{j}$ and $\zeta_{j}$ are indicator variables for $c_{1}$ after soft combining, and $c_{1}$ and $c_{2}$ using the proposed method, respectively. Furthermore, two gain measures are utilized. The total throughput gain $T G$ is computed as:

$$
T G=\left(\frac{C_{\text {prop }}}{C_{\text {conv }}}-1\right) .
$$

Similarly, the retransmission throughput gain $R G$ is computed as:

$$
R G=\left(\frac{E\left\{k_{1} \sum_{j=1}^{N} \zeta_{j}+k_{2} \sum_{j=1}^{N} \psi_{j}\right\}}{E\left\{k_{1} \sum_{j=1}^{N} \eta_{j}\right\}}-1\right) .
$$

In order to quantify the performance, simulations are utilized. Rayleigh fading with additive white gaussian noise at the receiver are assumed where the channel independently fades from one (re)transmitted symbol to the other. We further assume that the channel quality during the transmission phase and retransmission phase is equal on average. $\mathrm{BCH}$ codes are utilized with several combinations of FEC 1 and FEC 2 where $n=31$. Finally, BPSK modulation is assumed for simplicity. 


\section{REsults AND Discussion}

\section{A. Throughput Gain Analysis}

Fig. 3 shows the percentage BLEP of the first transmission (dashed lines) in addition to the percentage total throughput gains (solid lines) whereas the retransmission throughput gains are shown in Fig. 4.

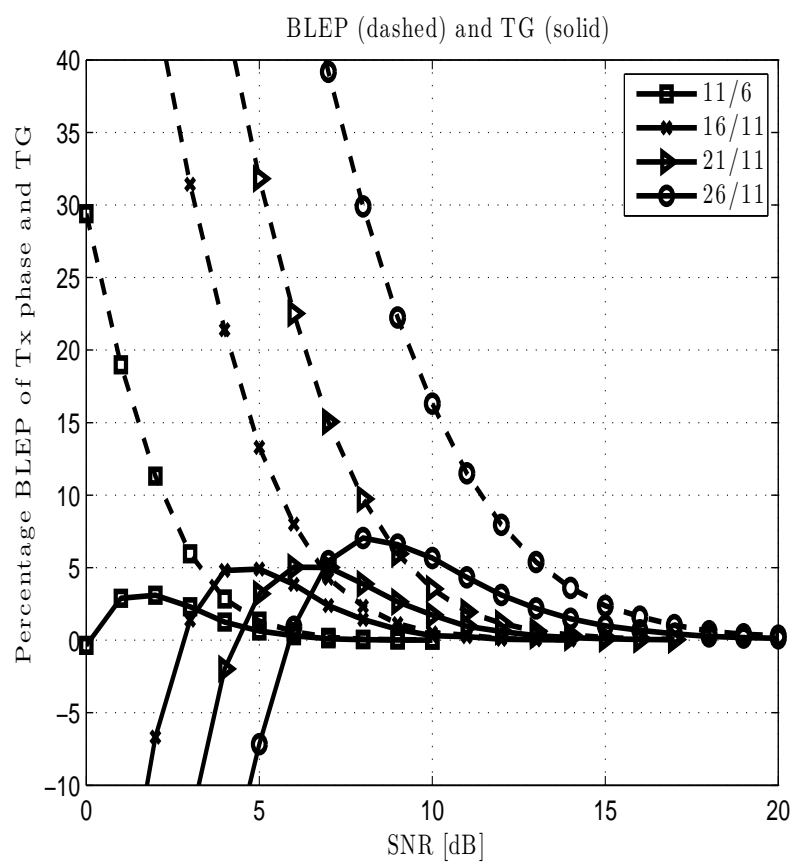

Fig. 3. Percentage total gain of the proposed scheme and percentage BLEP after the first transmission for four different combinations of FEC 1 and FEC 2.

The BLEP and gains are plotted in the same curve, both using a percentage scale, to easily observe at which BLEP the gains are obtained. The legends in both figures are presented as $k_{1} / k_{2}$ where e.g. $11 / 6$ refers to: $\mathrm{FEC} 1=(31,11,11)$ and FEC 2 $=(31,6,15)$. These results are based on the receiver structure in Fig. 2 using two iterations as described above.

From a practical point of view, best effort traffic can tolerate a BLEP up to $30-40 \%$, web browsing a BLEP of about $10 \%$, and delay-sensitive traffic a BLEP in the region of $1 \%$. As such one can divide the SNR range for a certain FEC 1 into three different regions based on the initial transmission BLEP. In Region I, the BLEP is too high for FEC 1 to be used in practice, and a code with lower code rate should be utilized. This can be seen in Fig. 3 for e.g. FEC $1=(31,21,5)$ at an SNR below $4 \mathrm{~dB}$

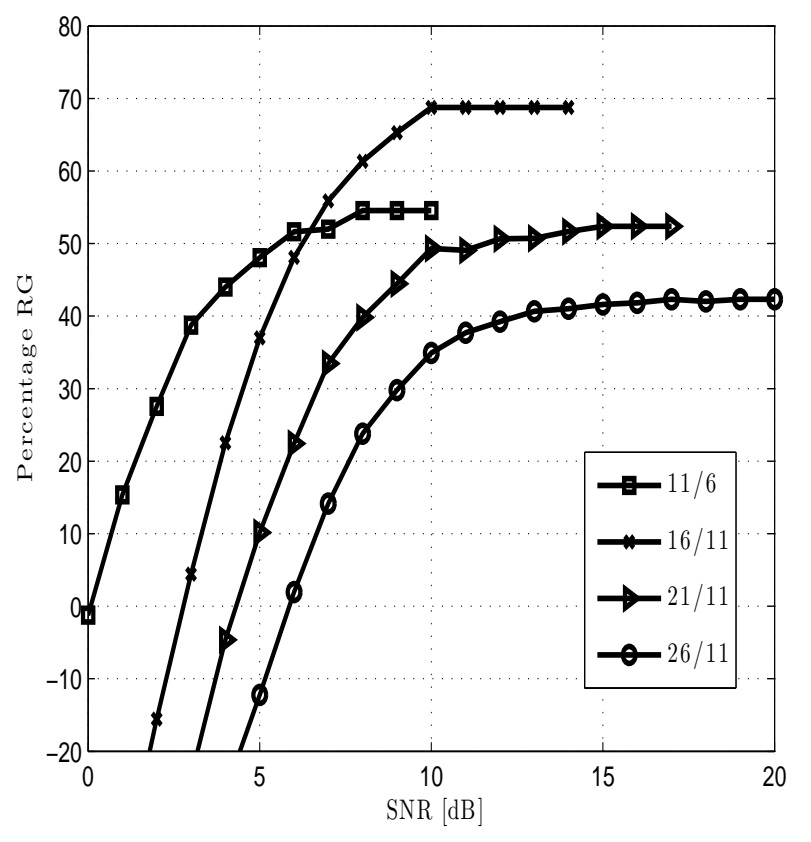

Fig. 4. Percentage retransmission gain of the proposed scheme for four different combinations of FEC 1 and FEC 2.

where it becomes more useful to utilize e.g. $(31,16$, 7 ) instead. In Region II, the BLEP is ranging from around $40 \%$ to around $5 \%$ which represents a region of practical interest for rather elastic traffic that can tolerate the delay from retransmissions. This corresponds to e.g. FEC $1=(31,21,5)$ at an SNR between 4 and $9 \mathrm{~dB}$. In Region III, the code is very robust as it has a BLER below $5 \%$ and is used for applications where retransmissions are very costly and undesirable, e.g. in systems that require too low latency. This corresponds to e.g. FEC $1=(31$, $21,5)$ at an SNR higher than $9 \mathrm{~dB}$. By examining Fig. 3 and Fig. 4, it can be seen that the proposed method is able to outperform conventional HARQ over all regions of practical interest (i.e. Region II and Region III) for all the studied FEC 1 and FEC 2 combinations.

In Region I, many retransmissions are necessary due to the very high BLEP (i.e. since BLEP is directly proportional to the number of retransmissions). For such a high BLEP, a more robust code compared to FEC 1 should be utilized. On the other hand, the BLEP in regions II and III is in line with what can be tolerated in practical systems. In Region II, it can be seen that the total number of retransmissions is still significant which means that 
the proposed method can be applied more often and that total throughput gains, not only retransmission gains, can be achieved. Total system throughput gains up to $7.75 \%$ can be achieved compared to a system using conventional HARQ with CC (see 26/11 in Fig. 3). The retransmission throughput gain increases as the SNR increases due to the fact that the number of errors in $e_{1}$ and $\boldsymbol{e}_{\boldsymbol{x}}$ decreases, leading to more correct decoding instances at the receiver. In Region III, the amount of retransmissions is significantly lower as the BLEP of the initial transmission is below $5 \%$. Such a low BLEP is typically required for delay-sensitive applications. In this region, it is of significant advantage to provide a method where retransmissions are able to contain more than just the failed codeword as this would effectively decrease the latency incurred from a retransmission for the remaining packets at the transmitter waiting to be transmitted. Another interesting observation that can be seen from Fig. 4 is that the proposed scheme is able to always achieve its maximum RG in Region III. The maximum retransmission gain $R G_{\text {max }}$ can be computed by substituting $\zeta_{j}, \psi_{j}$ and $\eta_{j}$ by 1 in (6) and resulting in:

$$
R G_{\max }=\left(\frac{k_{1}+k_{2}}{k_{1}}-1\right)
$$

which is dependent on the choice of FEC 1 and FEC 2 . For the simulated cases, the highest RG obtained is $68.75 \%$ which corresponds to FEC 1 and FEC 2 of $(31,16,7)$ and $(31,11,11)$ respectively. Note that $R G_{\max }$ is achieved when all retransmissions using the proposed method are decoded correctly (i.e. $\psi_{j}=\eta_{j}=1$ ). This typically happens at high SNR regions where retransmissions are mostly caused by instantaneous fading dips such that it is sufficient to retransmit the information without utilizing any combining at the receiver while still decoding correctly. This implicitly means that a retransmission with soft combining would be correctly decoded (i.e. $\zeta_{j}=1$ ). When no combining is needed in the retransmission phase, it is more beneficial to send new information combined with the old information as this would lead to a better exploitation of the good channel quality.

\section{B. Gains from the Iterative Approach}

With the results in Fig. 3 and Fig. 4 being based on utilizing an additional iteration compared to the non-iterative structure shown Fig. 2, an interesting aspect to examine is how much of the gains are achieved when less advanced processing is utilized. This is shown separately for $\boldsymbol{b}_{1}$ and $\boldsymbol{b}_{\mathbf{2}}$ in Fig. 5 and Fig. 6 respectively and assuming FEC $1=(31,21$, 5) and FEC $2=(31,11,11)$ for illustration.

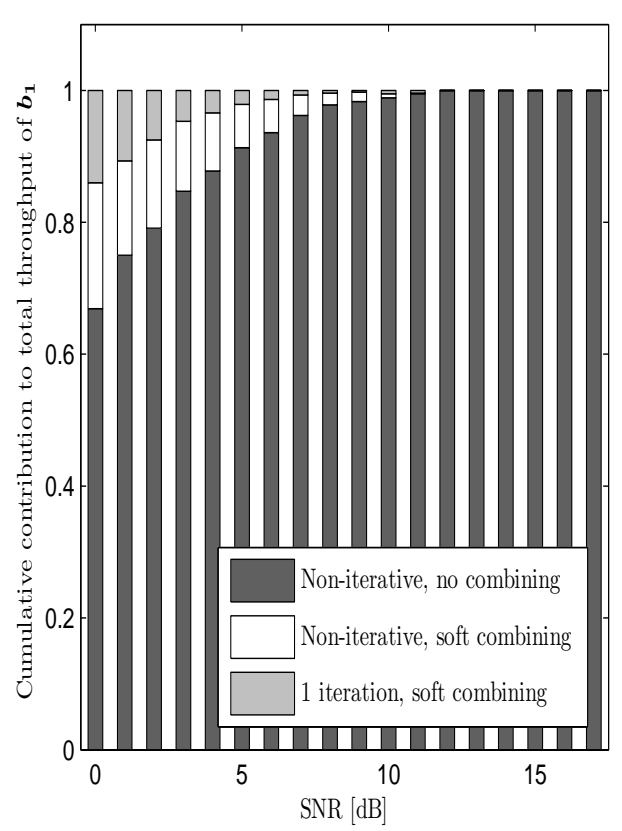

Fig. 5. Contribution of the different processing steps towards the total throughput of $\mathbf{b}_{1}$ assuming $\operatorname{FEC} 1=(31,21,5)$ and FEC $2=$ $(31,11,11)$.

For the case of $\boldsymbol{b}_{1}$, three different levels of complexity are considered: Non-iterative, no combining where the MRC, channel weighting and intermediate MOD and DEM blocks in Fig. 2 are disabled, i.e. no combining is used and $\hat{b_{1}}$ is solely based on $\hat{c_{1}}$, Non-iterative, soft combining which corresponds to the receiver structure as shown in Fig. 2, and 1 iteration, soft combining which corresponds to the results shown in Fig. 3 and Fig. 4. It can be seen that most of the gains from a second iteration are obtained over SNR regions where the codes utilized are not robust enough to be used in practice (i.e. Region I). On the other hand, the gains from soft combining for the proposed method are useful when operating in Region II, i.e. where combining would provide an estimate of $c_{1}$ with an enhanced quality to be decoded. However, these gains become very minimal when operating in Region III meaning that the receiver can safely disable the soft combining 


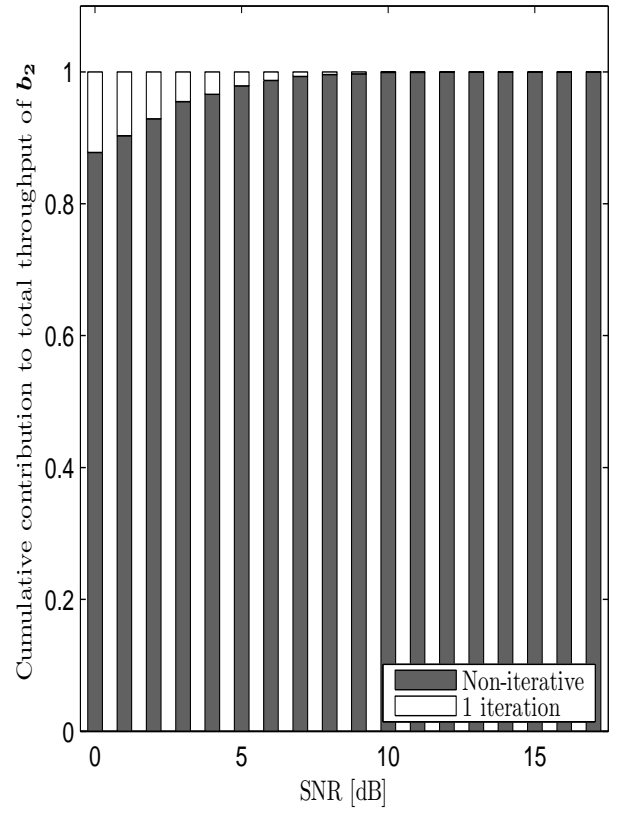

Fig. 6. Contribution of the different processing steps towards the total throughput of $\mathbf{b}_{\mathbf{2}}$ assuming $\mathrm{FEC} 1=(31,21,5)$ and FEC $2=$ $(31,11,11)$.

of $r_{1}$ and $r_{1}^{\prime}$ and simply use $\hat{c_{1}}$ for obtaining $\hat{b_{1}}$ thus decreasing the processing complexity.

For the case of $\boldsymbol{b}_{2}$, two different levels of complexity are considered, Non-iterative and 1 iteration. It can be seen from Fig. 6 that an iterative process is not needed for the BLEP regions of interest as most of the gains are already achieved due to FEC 2 being more robust than FEC 1 .

\section{Future Work}

As a future work, we are studying the generalization of the proposed algorithm to the case of multiple retransmissions where two main approaches have been identified. The first approach consists of applying the proposed method to all subsequent retransmissions (i.e. the second retransmission would add yet another new bit stream $b_{3}$ coded with FEC 3 , and so forth). The second approach consists of switching to conventional Chase combining or incremental redundancy in case the first retransmission using the proposed approach does not yield a correct decoding at the receiver (i.e. the second and subsequent retransmissions, if any, would retransmit either $c, c_{1}$ or $c_{2}$ ).

\section{CONCLUSiON}

This paper presented a novel scheme for enabling the combining of new data bits with retransmitted bits for unicast retransmissions in order to increase the system and user throughput. The new data bits are encoded with a smaller code rate than that of the initial bits in order to guarantee their ability to correct not only errors imposed on them from the channel, but also from the errors that occurred to the initial transmission of the retransmitted bits. A transmitter/receiver structure and data processing chain have been presented, and simulation results showed total throughput gains up to $7.75 \%$ and retransmission throughput gains of $68.75 \%$ for the utilized codes compared to HARQ with Chase combining.

\section{ACKNOWLEDGMENTS}

The authors would like to thank the anonymous reviewers for their valuable comments and suggestions to improve the quality of this manuscript.

\section{REFERENCES}

[1] D. Chase, "Code combining-a maximum-likelihood decoding approach for combining an arbitrary number of noisy packets," Communications, IEEE Transactions on, vol. 33, no. 5, pp. 385 - 393, May 1985.

[2] E. Dahlman, S. Parkvall, J. Skold, and P. Beming, 3G Evolution: HSPA and LTE for Mobile Broadband. Elsevier Science, 2008.

[3] P. Frenger, S. Parkvall, and E. Dahlman, "Performance comparison of HARQ with Chase combining and incremental redundancy for HSDPA," in Vehicular Technology Conference, 2001.

[4] P. Larsson and N. Johansson, "Multi-user ARQ," Vehicular Technology Conference, 2006.

[5] D. Nguyen, T. Tran, T. Nguyen, and B. Bose, "Wireless broadcasting using network coding," Vehicular Technology, IEEE Transactions on, vol. 58, pp. 782-786, Feb. 2009.

[6] Z. Li, Q. Luo, and W. Featherstone, "N-in-1 retransmission with network coding," Wireless Communications, IEEE Transactions on, vol. 9, no. 9, pp. 2689 -2694, Sep. 2010.

[7] S. Yun, H. Kim, and K. Tan, "Towards zero retransmission overhead: A symbol level network coding approach to retransmission," Mobile Computing, IEEE Transactions on, vol. 10, no. 8, pp. $1083-1095$, Aug. 2011.

[8] S.-Y. Li, R. W. Yeung, and N. Cai, "Linear network coding," IEEE Trans. Information Theory, 2003. 